\title{
Settling in Aggregating Colloidal Suspensions
}

\author{
D. Senis ${ }^{1}$, L. Talini ${ }^{1 *}$ and C. Allain ${ }^{1}$ \\ 1 Laboratoire FAST, Bât. 502, Campus universitaire, 91405 Orsay Cedex - France \\ e-mail: talini@fast.u-psud.fr \\ * Corresponding author
}

Résumé - Agrégation et sédimentation dans les suspensions colloïdales - Dans une suspension colloïdale où les particules s'agrègent, il existe une gamme de fractions volumiques pour laquelle la suspension forme un gel si fragile qu'il se tasse sous son propre poids. Nous présentons une étude de la cinétique de sédimentation d'un gel colloïdal formé par une suspension de particules de carbonate de calcium dans l'eau. La fraction volumique des particules a été systématiquement variée et différents régimes de sédimentation ont été observés. Les résultats expérimentaux sont décrits en utilisant un modèle 1D qui prend en compte à la fois la structure microscopique du gel colloïdal, assimilé à un empilement compact d'agrégats fractals, et ses propriétés mécaniques. L'angle d'inclinaison de la cellule par rapport à la verticale a également été varié et nous montrons que l'inclinaison peut induire l'apparition de nouveaux régimes de sédimentation. Les résultats, qui correspondent à une large gamme de conditions expérimentales, sont résumés dans un diagramme représentant les variations de $\Phi$ et $\alpha$. L'influence de l'inclinaison de la cellule a pu être décrite par un modèle 2D utilisant les résultats du modèle 1D introduit précédemment, ainsi que les arguments du modèle PNK (Ponder, Nakamura et Kuroda), classiquement utilisé pour décrire la sédimentation de suspensions de particules macroscopiques dans une géométrie inclinée. La description proposée est non seulement en très bon accord avec les résultats expérimentaux mais permet également de prédire le comportement de ce type de suspensions colloïdales, qui sont utilisées dans de nombreux processus industriels.

Mots-clés : gel colloïdal, sédimentation, effet Boycott, agrégats fractals.

Abstract - Settling in Aggregating Colloidal Suspensions - Strongly-aggregating colloidal suspensions form gels whose stability depends on the volume fraction $\Phi$ of the particles. In a given range of volume fractions, the formed gel is so fragile it collapses under its own weight. We present a study of the settling kinetics of such a colloidal gel. Systematic experiments have been performed in calcium carbonate aqueous suspensions of varying volume fractions. Different settling regimes have been observed and are successfully described using a $1 D$ model that takes into account both the microscopic structure of the gel constituted by a packing of fractal aggregates and its specific mechanical properties. We have also varied the inclination angle $\alpha$ of the cell to the vertical direction, which can lead to the occurrence of new settling regimes. The experimental results are summarised in a $\alpha \mathrm{vs}$. $\Phi$ diagram that covers a large range of experimental conditions. Using both our $1 D$ model and arguments similar to the ones used for macroscopic suspensions (PNK-Ponder, Nakamura and Kuroda-model), we propose a $2 D$ description of the influence of inclination that is in good agreement with the experimental data. The model we propose therefore enables both to interpret and predict the settling behaviours of stronglyaggregating colloidal suspensions that are involved in many industrial processes.

Keywords: colloidal gel, settling, Boycott effect, fractal aggregates. 


\section{INTRODUCTION}

Colloidal suspensions are involved in many practical applications for which their stability is crucial. Separation of the solid particles from the fluid depends on the external forces exerted on the suspension but also on the nature of the interactions between particles at a microscopic scale. Here, we consider the frequently encountered case of stronglyaggregating colloidal suspensions in which the particle density is large compared to the fluid density and that can therefore separate when submitted to gravity.

We have shown in previous works $[1,2]$ that the coupling between settling and aggregation in such suspensions leads to distinct behaviours depending on the volume fraction of the particles, $\Phi$. As illustrated in Figure 1, when $\Phi$ is small $\left(\Phi<\Phi^{*}\right)$, deposition of individual aggregates is observed. At the beginning of the phenomenon, the cluster growth is solely controlled by Brownian diffusion. As their size becomes large enough, the clusters settle separately and deposit onto the bottom of the cell forming a loose sediment. Contrarily, when the volume fraction is large $\left(\Phi>\Phi^{* *}\right)$, no settling occurs; the suspension forms a stable colloidal gel, i.e. a close packing of aggregates filling the whole cell that does not separate under the action of gravity (Fig. 1). In the intermediate regime, when $\Phi^{*}<\Phi<\Phi^{* *}$, a gel forms very rapidly but this gel is fragile and collapses under its own weight. A sharp interface then separates a clear supernatant from the gel; this interface moves down until it reaches its equilibrium height.

In previous papers, we have presented an analysis of the equilibrium of such a gel in the intermediate regime [3,4]. We have shown that the fractal structure of the aggregates forming the gel leads to very specific properties. In particular, the mechanical response of the gel can be described using a yield compressive stress that is power law-dependent on the volume fraction. Moreover the relative volume occupied by the sediment $\Omega$, defined as $\Phi / \Phi_{s}$ ( $\Phi_{s}$ being the volume fraction in the sediment), is very large even at small volume fractions (typically $\Omega=0.4$ for $\Phi=0.5 \%$ ).

On account of its microscopic structure, the settling kinetics of such a colloidal gel is expected to strongly differ from the one observed in usual macroscopic suspensions constituted by non-Brownian particles. In order to study this kinetics, the first relevant parameter to be varied is obviously the volume fraction $\Phi$. Inclining the settling cell to the direction of gravity may also lead to original behaviours, the influence of the inclination angle $\alpha$ of the cell is therefore important to consider from both fundamental and practical points of view. In the case of macroscopic suspensions, it is now well established that inclination of the settling cell to the vertical direction causes a strong enhancement of the settling velocity. This effect, known as Boycott effect, results from the formation of a thin slit of particle-free fluid along the upper sidewall of the cell and is classically described using

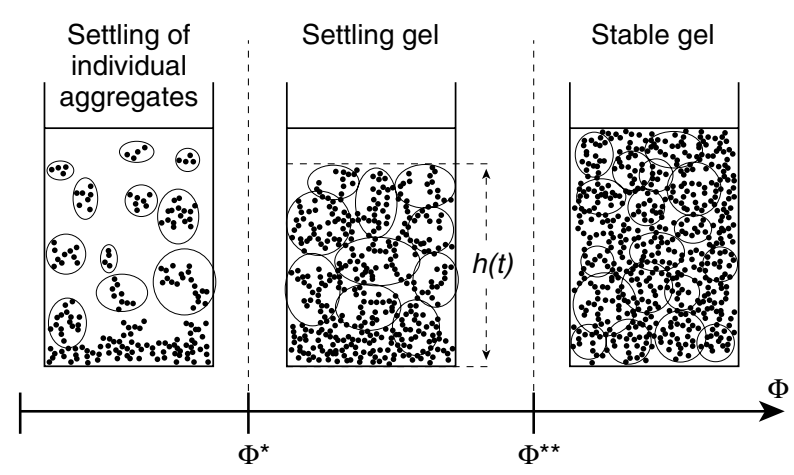

Figure 1

Schematic representation of the behaviour of a stronglyaggregating colloidal suspension submitted to gravity as a function of the volume fraction of the particles.

the Ponder, Nakamura and Kuroda (PNK) model $[5,6]$. In the case of a settling colloidal gel, one expects that the specific properties of the gel modify the effects of inclination.

In this paper we present a synthetic view of the different types of behaviour encountered with a strongly-aggregating suspension. Systematic experiments have been performed in a large range of volume fractions and inclination angles of the cell. The experimental results are interpreted by $1 \mathrm{D}$ or $2 \mathrm{D}$ models based on a description that considers the gel structure and the different forces in presence. The developed models not only provide a good description of the experimental data but also enable to predict the different settling regimes that are observed. The somewhat complex behaviours that appear either in vertical and inclined cells are thus described using only one parameter, which is the fractal dimension $D$ of the aggregates. The paper is organised as follows: in Section 1 we present the preparation of the suspensions as well as the method of measurement. Section 2 is devoted to both the experimental results and the models we propose to describe them.

\section{MATERIALS AND METHODS}

\subsection{Suspensions}

The experiments were carried out with aqueous calcium carbonate suspensions. The particles (Socal U1 supplied by Solvay Co.) are monodisperse and almost spherical and have a good chemical purity. Their radius is $a=35 \mathrm{~nm}$ and their density is $\rho=2.7 \mathrm{~g} / \mathrm{cm}^{3}$. Once in water, the surface charge borne by the particles strongly depends on the concentration of calcium and carbonate ions. The experimental conditions (free atmosphere, $\left[\mathrm{Ca}^{2+}\right] \cong 10^{-4} \mathrm{~mol} \cdot \mathrm{l}^{-1}, \mathrm{pH}$ ranging from 8.7 to 9.2) are such that this charge is very small and the net colloidal interaction reduces to the Van der Waals attraction. 
The samples are prepared as follows: calcium carbonate powder is added to deionised water and the obtained suspensions are strongly stirred during two weeks. Systematic measurements of $\Omega$, the relative volume occupied by the sediment at equilibrium, have been performed prior to each experiment and showed that the suspensions are well dispersed for $\Phi \leq 1 \%$ [7]. Practically, the results presented in the following correspond to particles volume fractions ranging from $0.3 \%$ to $1 \%$ (varying every $0.1 \%$ ) which corresponds to the intermediate regime $\left(\Phi^{*}<\Phi<\Phi^{* *}\right)$.

\subsection{Measurements of the Interface Height}

The experimental cells made of Plexiglas are either cylindrical (inner diameter $12 \mathrm{~mm}$ ) or of rectangular section (internal depth and width respectively $30 \mathrm{~mm}$ and $d=12 \mathrm{~mm}$ ). In both cases, the useful height is $H=70 \mathrm{~mm}$. Measurements at equilibrium were performed in cylindrical cells whereas the settling kinetics was studied in rectangular cells which enabled us to work with 2D models. In the following we denote $h(t)$ the height of the interface between the supernatant and the settling gel (Figs. $2 a$ and $2 b$ ). Before each experiment, the cells are completely filled with suspension and carefully sealed in order to avoid the presence of a meniscus at the top of the suspension (see [8] for more details). For the experiments in an inclined cell, the cell is tilted at an angle $\alpha$, the axis of rotation being parallel to the largest side of the cell (Fig. 2b). Practically, $\alpha$ ranges from $5^{\circ}$ to $45^{\circ}$ and has been varied every $5^{\circ}$. The uncertainty on the measurement of the angle is $0.3^{\circ}$.
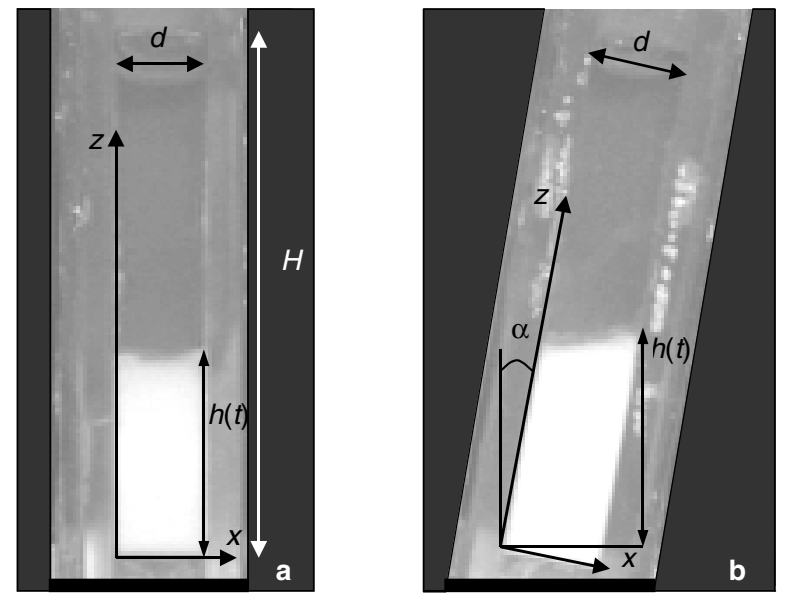

Figure 2

Photographs of the small side (width $d=12 \mathrm{~mm}$ ) of rectangular settling cells filled with a suspension of volume fraction $\Phi=0.5 \%$. The colloidal gel appears in white. The cell may stand vertical (a) or tilted at an angle $\alpha$ (b). The height of the interface between the gel and the supernatant is denoted by $h(t)$.
We only briefly describe the data acquisition process since it is detailed elsewhere [7,8]. The motion of the interface is recorded through a video camera and space $v s$. time diagrams are constructed from the recordings. These diagrams are eventually digitised in order to extract the position of the interface as a function of time $h(t)$. In order to determine $h(t)$ with a good accuracy, special care is taken to ensure the horizontality of the optical axis as well as a good optical contrast of the recordings. The uncertainty $\Delta h$ is of the order of $0.3 \mathrm{~mm}$. It should be noted that for the sake of readability only a small fraction $(20 \%)$ of the experimental points is displayed in the $h(t)$ plots shown in the following.

\section{KINETICS OF SETTLING}

We have systematically studied the settling of the suspension according to the values of the tilt angle $\alpha$ and of the volume fraction $\Phi$ within the intermediate volume fraction regime. The experimental results are summarised in the $\alpha v s$. $\Phi$ diagram of Figure 3: each of the three regions corresponds to the succession of different settling regimes that are detailed in the following as well as their theoretical descriptions. Note that for volume fractions larger than $1 \%$ the dispersion of the suspensions is poor and the different regimes can hardly be distinguished because of very small displacement of the interface. For volume fractions smaller than $0.3 \%$, settling by cluster deposition is observed (the gel does not form).

The presentation of the different regimes in what follows remains qualitative since the aim of the present work is to give an overall view of the matter. The reader should report to previous papers [7-9] to find quantitative results.

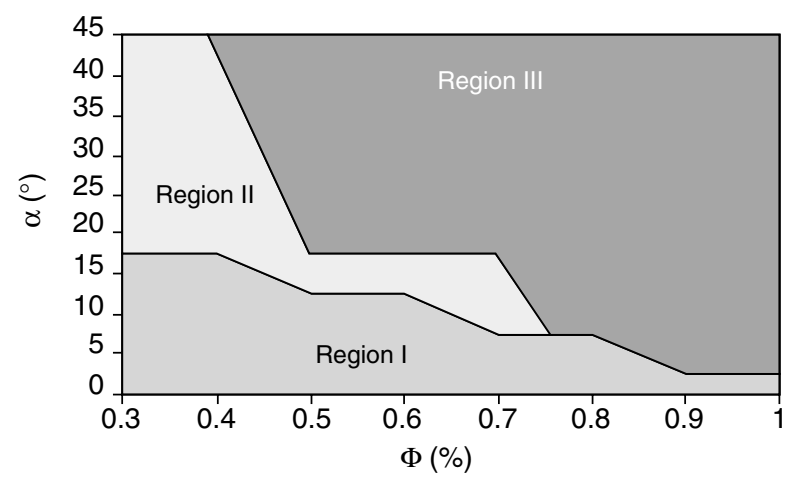

Figure 3

$\alpha$ vs. $\Phi$ diagram. Between a slow constant velocity regime and a final compaction regime, different successive phenomena can govern the settling: only crack apertures (Region I), or Boycott effect due to cell inclination followed by crack apertures (Region II) or only Boycott effect (Region III). 


\subsection{Behaviour at Small Volume Fractions and Tilt Angles}

Region I of the diagram corresponds to relatively small volume fractions and tilt angles (including $\alpha=0$, i.e. a vertical cell). Typical variations of $h(t)$ observed in this region are displayed in Figure 4. Three different velocity regimes can be distinguished: after a slow displacement of the interface at a constant velocity $v$, a sudden acceleration of the interface height displacement occurs and is soon followed by a second linear domain of variation characterised by a velocity about ten times larger than $v$. Finally, the interface displacement slows down until the gel reaches its equilibrium height.

The end of the first settling regime coincides with the occurrence of eruptions at the interface between the gel and the supernatant. Figure 5 shows a photograph of one of these eruptions. We have found that these eruptions correspond to the aperture of fractures inside the gel and that these fractures form preferential paths of upward flow, thus allowing an increase in the settling velocity. The formation of these cracks as well as their influence on the settling are quite complex and are beyond the scope of the present work. They will be the object of a forthcoming paper.

We therefore focus on the other observed regimes of velocity. In these regimes, since $\alpha$ and therefore the influence of inclination are small, we consider that no transversal compaction of the gel occurs, i.e. that the volume fraction remains constant in the direction perpendicular to the $z$ axis. The settling regimes can therefore be described using a 1D model provided the inclination angle is small enough. The balance of the stresses exerted on a slice of gel located at height $z$ and in which the volume fraction is $\varphi(z)$ writes:

$$
\Delta \rho \varphi(z) \mathrm{g} \cos \alpha=-\frac{\partial \sigma}{\partial z}+F+\Sigma
$$

where $\Delta \rho$ represents the difference of density between the particles and the suspending fluid, $g \cos \alpha$ the effective gravity when the cell is tilted at an angle $\alpha, \sigma$ the stress at height $z$. $\Sigma$ results from the hydrodynamic viscous friction between the gel and the solvent and $F$ from the solid friction between the gel and the cell walls.

The different terms of Equation (1) indeed need further determination which can be done by considering the dominant actions exerted on the gel according to the different time scales:

- at short times, the compaction of the gel is negligible in the whole gel except near the bottom of the cell. The stress contribution $\partial \sigma / \partial z$ is therefore very small compared to the hydrodynamic friction and the volume fraction can be considered as uniform and taken equal to the initial volume fraction of suspension, $\Phi$. The influence of the cell width on the settling being experimentally very weak at short times, the friction of the gel with the cell walls can be neglected. Equation (1) thus reduces to a balance between the gravitational forces and the hydrodynamic friction. Furthermore, $\Sigma$ can be evaluated by describing the gel as a close packing of aggregates that are impenetrable to the flow. In the particles frame, the gel is then analogous to a porous medium whose permeability can be related to the microscopic structure of the aggregates [8].

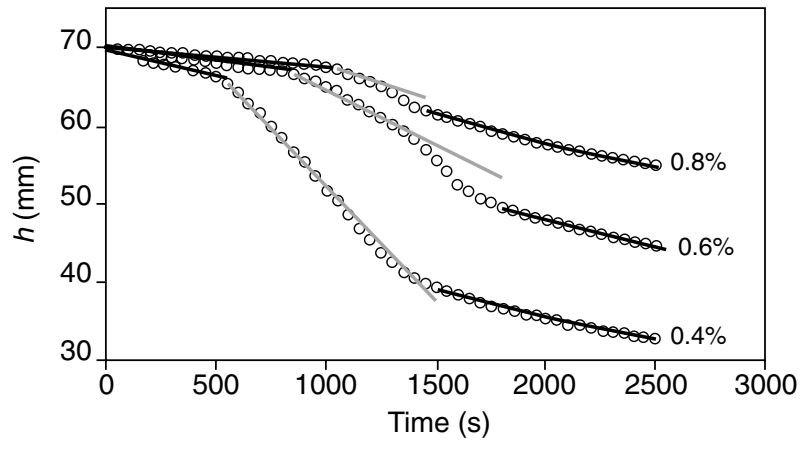

Figure 4

Interface height as a function of time of calcium carbonate suspensions of different volume fractions in a vertical cell. Three different settling regimes can be distinguished from the experimental data (circles). The full black lines respectively correspond to the predictions of the 1D model for the first and third regimes, the grey line to the linear domain of the second regime.

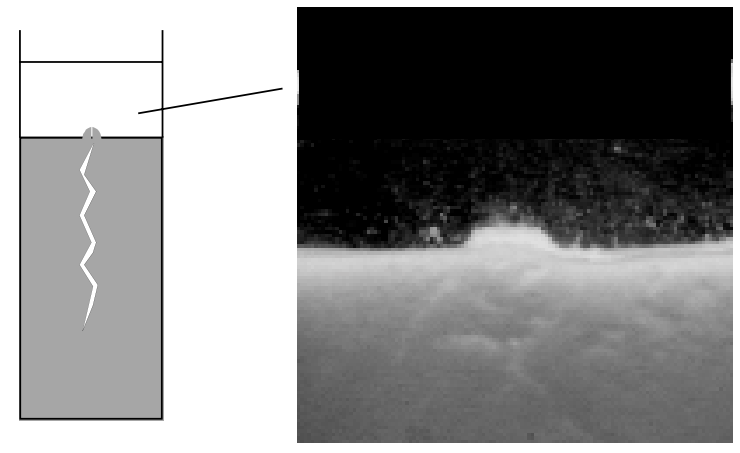

Figure 5

Schematic representation of a crack in the bulk of the settling suspension. The crack provides a path for the backflow of the fluid and causes a small "eruption" at the interface between the supernatant and the settling gel. A close view of such an eruption is shown in the photograph whose width corresponds to $10 \mathrm{~mm}$. 
Finally, accordingly to the experimental results, the settling velocity is constant and scales as:

$$
v=A \cos ^{2} \alpha \Phi^{(1-D) /(3-D)}
$$$$
\text { with: } \quad A=\frac{\Delta \rho a^{2} \mathrm{~g}}{45 \eta}\left[\frac{1-\varphi_{e}^{3}}{\varphi_{e}^{2(2-D) /(3-D)}}\right]
$$

where $\eta$ is the solvent viscosity, $a$ the particles radius, $\varphi_{e}$ the close-packing volume fraction $\left(\varphi_{e} \approx 0.63\right)$ and $D$ the fractal dimension;

- in the third velocity regime that follows the crack apertures regime, the cracks heal up and the settling can again be described using a 1D model (Eq. (1)). In the frame of this model, the solid friction with the cell walls can still be neglected. In this case however the volume fraction $\varphi(z)$ is no more constant: $\varphi(z) \neq \Phi$. Assuming that compaction is uniform along the gel height simply yields to $\varphi(z)=\bar{\varphi}(t)=\Phi H / h(t)$. Contrarily to the preceding case, the gel compaction is important and the stress contribution can no longer be neglected along the whole height of the gel. However, close to the top of the suspension (i.e. at $z \approx h(t)$ ), the backflow fluid velocity remains large. At this height, the hydrodynamic friction therefore still rules the settling and once again the stress term in Equation (1) can be neglected. Resolution of Equation (1) is performed assuming as previously that the gel can be described as a close packing of spheres impenetrable to the solvent flow (in this case however the radius of the aggregates is a function of time since compaction takes place by internal brittle fracture of the aggregates). The resulting settling velocity is no more constant but shows the same dependency on the volume fraction since $\mathrm{d} h / \mathrm{d} t=-K(\Phi / h)^{(1-D) /(3-D)}$. The prefactor $K$ depends on the same parameters as the prefactor $A$ (Eq. (2)) and also on the height of the cell $H$;

- at long times $(t \rightarrow \infty)$, the gel reaches equilibrium and the hydrodynamic friction vanishes. At equilibrium the gravitational forces are balanced by the stress contribution and the solid friction with the walls, $F$. The stress contribution is described introducing a yield compressive stress that depends on the volume fraction. Assuming furthermore that the friction between the gel and the walls is of the same type than a solid/solid friction enables to calculate $F$ following the classical laws used for solid friction [10]. Resolving Equation (1) thus enables to predict not only the value of the sediment height but also the volume fraction $\Phi^{* *}$ above which the gel is stable [3, 4].

Although the presented 1D model is very simple, the agreement between the experimental data and the theoretical predictions has proved to be excellent within ranges of volume fractions and tilt angles corresponding to Region I (see Fig. 4 as an example). In the large range of volume fractions and inclination angles investigated, all the settling data could be described using only one adjusted parameter, i.e. the fractal dimension $D$. The corresponding value of $D(2.43 \pm 0.04)$ is very close to former measurements performed with the same calcium carbonate suspensions by a different method [11] (i.e. $D=2.40 \pm 0.05$ ). This agreement justifies the assumptions made on the gel structure as well as on the nature and on the relative importance of the phenomena governing the settling.

\subsection{Behaviour at Large Volume Fractions and Tilt Angles}

The settling behaviour is modified when the inclination angle is increased and at large enough volume fractions (Regions II and III of the $\alpha v s$. $\Phi$ diagram). This modification is the consequence of the apparition of an effect analogous to the Boycott effect that is classically observed in macroscopic suspensions. As pointed out in the introduction, this effect is due to the formation of a clear fluid slit along the upper wall of the cell that creates a path of upward flow for the suspending fluid. The formation of this particle-free slit results in an increase of the settling velocity. This effect can be simply described by the PNK model that consists in calculating the total volume of clear fluid formed by time unit by adding two contributions: one corresponds to the backflow due to the settling in the $z$ direction and the other to the backflow of suspending fluid feeding the slit $[5,6]$. In the frame of the PNK model, the settling velocity is then given by:

$$
\frac{\mathrm{d} h}{\mathrm{~d} t}=-v\left(\cos ^{2} \alpha+\sin \alpha \frac{h(t)}{d}\right)
$$

where $v$ is the velocity in a vertical cell and $d$ the cell width.

We have shown in a previous paper [9] that a similar Boycott effect could take place in an aggregating colloidal suspension even though it differs by its microscopic structure. A clear fluid slit can form between the upper inclined wall and the gel, which leads to an increase of the settling velocity. However, the slit can form only if the stress in the transverse direction exceeds the yield stress of the gel, i.e. only if the inclination angle is large enough, which explains the absence of inclination influence at relatively small angles (Section 2.1). Moreover, contrarily to usual suspensions, the formation of the slit is not instantaneous but takes the time needed for the compaction of the gel in the transverse direction. Another difference with usual suspensions is that, once the clear fluid slit has formed, the presence of the voluminous sediment (i.e. the compacted gel in the bottom of the cell) can decrease the length of the slit and therefore reduce the enhancement of the settling velocity.

To summarise the influence of inclination upon the settling, let us note that the qualitative description of Section 2.1 remains valid whatever the inclination angle. At short times, the settling velocity is still constant and relatively weak since 
the slit has not formed yet. At the longest time scales, the clear fluid slit has vanished as a consequence of the gel compaction and a 1D model (Eq. (1)) describes the settling as well as the value of the sediment volume. As illustrated in Figure 6, the agreement with experimental data is excellent. At intermediate times, the settling differs from the one observed at small angles by the occurrence of a new regime succeeding the first constant velocity regime. This regime during which the interface accelerates is the consequence of the formation of the clear fluid slit as confirmed by visual observation. Contrarily to the other regimes, the gel is no more homogeneous in the transverse direction and a $2 \mathrm{D}$ model is required to describe it. To that extent we have used arguments similar to the ones of the PNK model combined with the theoretical description of the gel settling in a vertical cell. Taking into account the mechanical properties of the gel, we have thus been able to accurately predict the occurrence of this regime and the corresponding settling velocity. As pointed out in the last paragraph, the presence of the voluminous sediment can influence the settling and lead to a decrease of the velocity. The complete description and prediction of the different regimes is quite complex and the reader should refer to a previous paper to find a more detailed study [9]. Let us note that experimentally, the occurrence of the different regimes as well as the interface displacements are in a very good agreement with the developed model (Fig. 6). Furthermore, the only adjusted parameter is the fractal dimension $D$ of the aggregates. The corresponding value of $D$ coincides with the one obtained in the case of small angles and volume fractions.

Finally, let us note that the difference between Regions II and III of the diagram is that in the conditions of Region III,

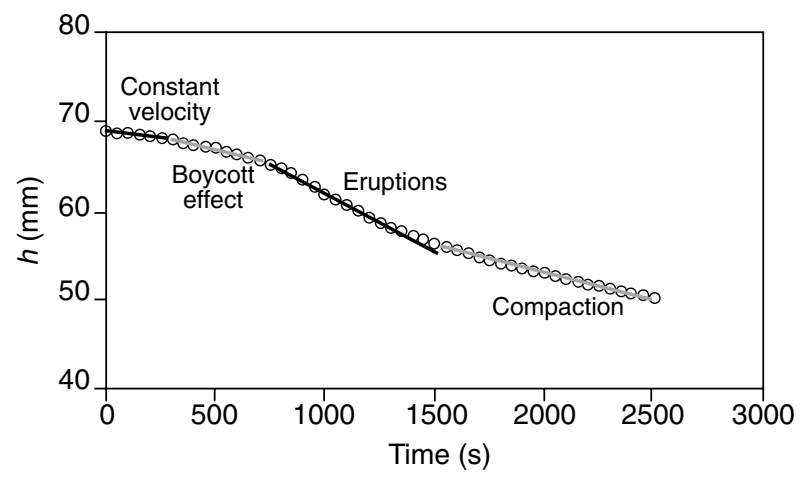

Figure 6

Interface height as a function of time for $\Phi=0.7 \%$ and $\alpha=10^{\circ}$. Besides the three velocity regimes occurring in a vertical cell, the inclination of the cell leads to a regime of enhanced velocity (Boycott effect). The experimental data (circles) is well described using the model we propose (full lines). no eruption due to crack aperture is observed during the settling whereas they succeed to the Boycott effect regime in Region II (Fig. 6). The regime governed by these fractures as well as their occurrence remains to be modelled and further experiments are under way, in particular to better understand the role of the solid friction of the gel with the cell walls. Note also that at very large tilt angles and volume fractions, the gel tends to stick to the lower wall of the cell (Fig. 7). In this case, a settling velocity can no more be defined and here again the influence of solid friction still requires to be further investigated.

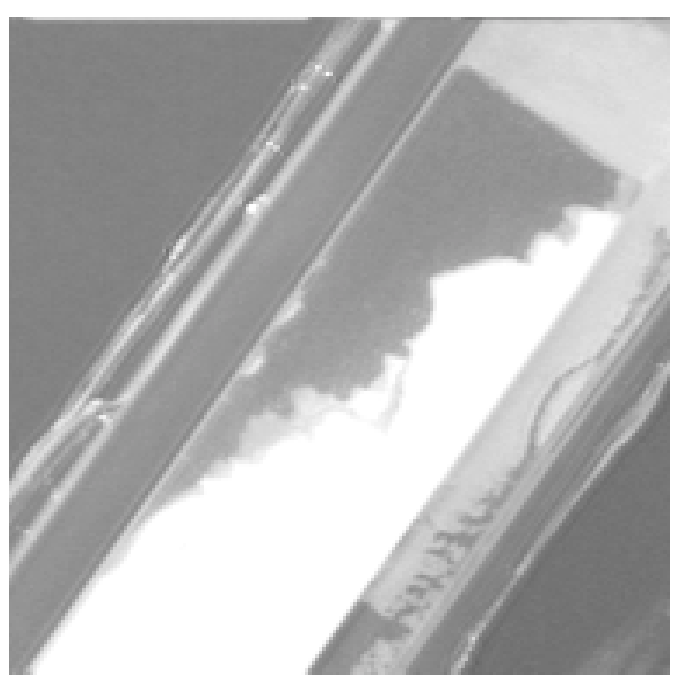

Figure 7

Photograph of the gel that has stuck to the lower sidewall of the cell during its settling. This phenomenon is observed at large volume fractions and tilt angles.

\section{CONCLUSION}

In conclusion, we have performed a systematic study of the settling behaviour of a strongly-aggregating colloidal gel according to the particles volume fraction as well as the inclination angle of the cell to the vertical. The experimental results are summarised in a $\alpha v s$. $\Phi$ diagram that provides a complete view of the different behaviours that can be encountered in such a suspension. Taking into account the gel microscopic structure, we have been able to interpret and describe all the different settling regimes in a vertical cell but one resulting from the aperture of cracks in the bulk of the gel. The experimental data show an excellent agreement with the proposed 1D model. In an inclined cell, new settling regimes are observed. The influence of the inclination angle is analogous to the Boycott effect well known in usual suspensions and classically described by the PNK model. To describe these settling regimes, we use a $2 \mathrm{D}$ model that 
derives from both the PNK model and the description of the gel properties that have been confirmed by the small angles investigations. Here again the agreement between theory and experiments is excellent.

\section{ACKNOWLEDGEMENTS}

We especially acknowledge J.F. Argillier for many fruitful discussions and we thank A. Audibert and IFP team for their interest in the subject. Laboratoire "Fluides, Automatique et Systèmes thermiques” is a laboratory of Universities Paris 6 and Paris 11, and of CNRS (UMR 7608). This work is part of the thesis of D. Senis, that was sponsored by the ministère de l'Enseignement supérieur et de la Recherche.

\section{REFERENCES}

1 Allain, C., Cloître, M. and Wafra, M. (1995) Aggregation and Sedimentation in Colloidal Suspensions. Phys. Rev. Lett., 74, 8, 1478-1481.

2 Allain, C. and Senis, D. (1997) Sédimentation et agrégation dans les suspensions colloïdales, in De la tectonique des plaques à la dynamique du tas de sable - Introduction aux suspensions en géologie et en physique, Iledefonse, B., Allain, C. and Coussot, P. (eds.), Cemagref, Paris.
3 Senis, D. and Allain, C. (1997) Scaling Analysis of Sediment Equilibrium in Aggregated Colloidal Suspensions. Phys. Rev. E, 55, 6, 7797-7800.

4 Senis, D. and Allain, C. (1997) Sedimentation and Compaction of Calcium Carbonate Aggregating Suspensions: Scaling Analysis of the Equilibrium. Oil \& Gas Science and Technology, 52, 2, 191-197.

5 Ponder, E. (1925) On Sedimentation and Rouleaux Formation. Quart J. Expt. Physiol., 15, 235-252.

6 Nakamura, H. and Kuroda, K. (1937) La cause de l'accélération de la vitesse de sédimentation des suspensions dans les récipients inclinés. J. of Med. College in Keijo, 8, 256296.

7 Senis, D. (1998) Agrégation, gélification et sédimentation dans les suspensions colloïdales et les fluides complexes. Thesis, University Paris 6.

8 Senis, D., Gorre-Talini, L. and Allain, C. (2001) Systematic Study of the Settling Kinetics in an Aggregating Colloidal Suspension. Eur. Phys. J. E, 4, 1, 59-68.

9 Senis, D., Gorre-Talini, L. and Allain, C. (2000) Settling in Aggregating Colloidal Suspension: Analysis of Cell Inclination Influence. Eur. Phys. J. E, 2, 1, 31-38.

10 Bowden, F.P. and Tabor, D. (1959) Le frottement et sa mesure, in Friction et Lubrification, Dunod, Paris.

11 Wafra, M. (1994) Sédimentation en présence d'agrégation dans les suspensions colloïdales : étude expérimentale des suspensions de carbonate de calcium. Thesis, University Paris 13

Final manuscript received in January 2001 